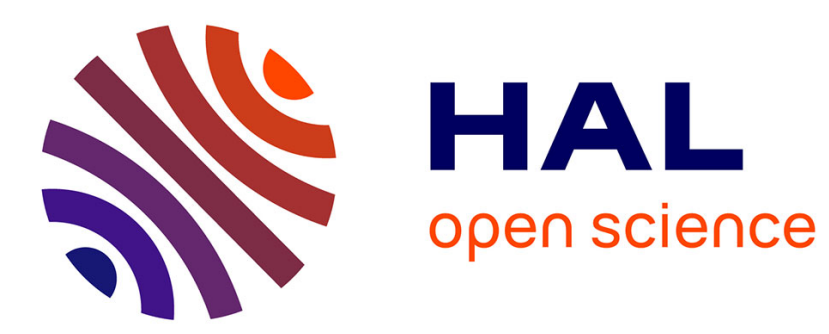

\title{
Feral horses synchronize their collective behavior at multiple levels of organization
}

Brenda Mccowan

\section{To cite this version:}

Brenda Mccowan. Feral horses synchronize their collective behavior at multiple levels of organization. 2021, pp.100001. 10.24072/pci.networksci.100001 . hal-03260228

\section{HAL Id: hal-03260228 \\ https://hal.science/hal-03260228}

Submitted on 14 Jun 2021

HAL is a multi-disciplinary open access archive for the deposit and dissemination of scientific research documents, whether they are published or not. The documents may come from teaching and research institutions in France or abroad, or from public or private research centers.
L'archive ouverte pluridisciplinaire HAL, est destinée au dépôt et à la diffusion de documents scientifiques de niveau recherche, publiés ou non, émanant des établissements d'enseignement et de recherche français ou étrangers, des laboratoires publics ou privés. 


\title{
Peer Community In Network Science
}

\section{Feral horses synchronize their collective behavior at multiple levels of organization}

\author{
Brenda McCowan based on reviews by Frédéric Amblard, Krishna \\ Balasubramaniam, Krishna Balasubramaniam and 1 anonymous reviewer
}

\section{A recommendation of:}

\author{
Behavioural synchronization in a multilevel society of feral horses \\ Tamao Maeda, Cedric Sueur, Satoshi Hirata, Shinya Yamamoto (2021), bioRxiv, \\ 2021.02.21.432190, ver. 3 peer-reviewed and recommended by Peer community in \\ Network Science. 10.1101/2021.02.21.432190
}

\section{Open Access}

Submitted: 23 February 2021, Recommended: 08 June 2021

Cite this recommendation as:

Brenda McCowan (2021) Feral horses synchronize their collective behavior at multiple levels of organization. Peer Community in Network Science, 100001. 10.24072/pci.networksci.100001

Published: 14 June 2021

Copyright: This work is licensed under the Creative Commons Attribution-NoDerivatives 4.0 International License. To view a copy of this license, visit

http://creativecommons.org/licen ses/by-nd/4.0/

\section{Recommendation}

In their article "Behavioural synchronization in a multilevel society of feral horses", Maeda and colleagues (2021) use stochastic multi-agent based modeling to explore the degree to which feral horses synchronize their behavior across multiple levels of organization. The authors compare a drone-derived empirical data set on a feral population of horses with simulated data from multi-agent-based models to determine whether behavioral synchronization of resting and movement states in a multilevel society can be described by one of three models: $A$ ) independent model in which horses do not synchronize, B) anonymous model in which horses synchronize with any individual in any unit, C) unit-level social model in which horses synchronize only within units and D) herd-level social model in which horses synchronize across and within units, but internal synchronization is stronger. In a series of 100 simulations for each of seven different models, the authors conclude that evidence for the herd-level model had the strongest support in relation to the empirical data. This finding suggests that connections among individuals in such multi-level societies are rather complex in that local connections are not the only interactions driving social behavior, and specifically synchronization. This approach could be successfully applied to a number of different species that exhibit multi-level organization and possibly fission-fusion dynamics.

This study is especially innovative and interesting for three major reasons. First, the use of drone technology to successfully identify individual animals and generate social networks is highly novel and permits the study of large multi-level social groups of animals that previously have been challenging to study due to limitations in collecting data at an appropriate scale. Second, the comparison of multi-agent-based models with actual empirical data is highly applauded. Most agent-based studies design their parameters from previous empirical studies, (sometimes with questionably simple 


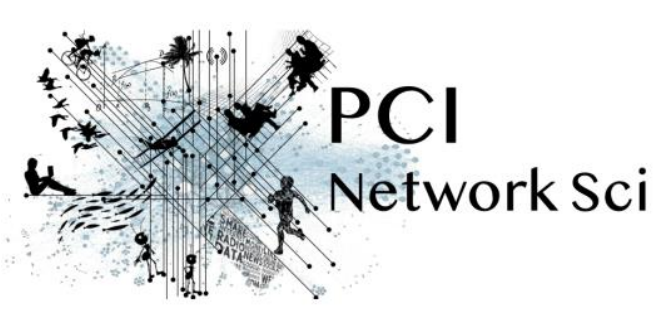

assumptions) but rarely do they actually compare model outputs to their own empirical data. This is an important next step in the burgeoning field of agent-based modeling. Finally, this study sheds light on the utility of using relatively simple mathematical models to explain highly complex behavior. It also highlights that feral horses can synchronize their behavior beyond clustered local connections which suggests that they possess the cognitive ability to track the behavior of individuals at higher social orders. As the authors state, in a multilevel society, inter-unit distance should be moderate, that is "not too close but not too far" because this strategy simultaneously avoids inter-unit competition while also providing the benefits of social buffering that comes with large group living, such as protection from bachelors or predators.

As the authors dutifully note, there were also some limitations to the study: (1) the relatively sparse empirical dataset that made it difficult to resolve the relative fitness of the two herd-level models (absolute versus proportional social models), (2) the lack of a temporal component that would provide a better understanding on how synchronization flows through the social/spatial network, and (3) the limited variation in the parameters tested which constrained identification of their true function in the model. Such limitations, however, provide fruitful avenues for further development of the model in future studies.

Overall then, this study provides new insights into the processes underlying the behavioral synchronization process and thus nicely contributes to the understanding of collective behaviors in complex animal societies as well as the evolution and functional significance of multi-level animal societies. This study is a fine addition to both the fields of agent-based modeling and the evolution of collective behavior in complex societies. I thus highly endorse its publication.

\title{
References
}

Maeda T, Sueur C, Hirata S, Yamamoto S (2021) Behavioural synchronization in a multilevel society of feral horses. bioRxiv, 2021.02.21.432190, ver. 3 peer-reviewed and recommended by Peer community in Network Science. https://doi.org/10.1101/2021.02.21.432190

\section{Reviews}

Toggle reviews

\section{Reviewed by Krishna Balasubramaniam, 2021-06-04 23:08}

I believe that the authors have done a great job of addressing the first round of comments from myself and the other reviewers. I have no further comments to add, and now recommend the manuscript for publication. Congratulations to the authors on a very interesting study!

\section{Revision round \#1}

\author{
2021-03-28
}

\section{Author's Reply}

Download author's reply (PDF file)

\section{Decision round \#1}

\author{
Dear Authors,
}

I have received three reviews for your preprint. All reviewers found your preprint very interesting and wellwritten. Yet each of them also provided suggestions that will enhance the quality of your article. Please take 


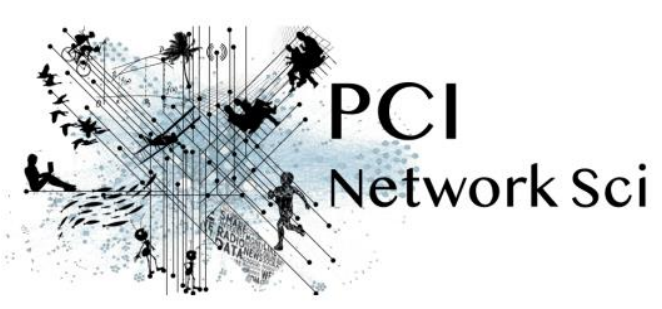

these comments into consideration in your resubmission. Also please carefully review for appropriate grammar and typos throughout your preprint.

Thank you for choosing PCI Network Science for your preprint. I look forward to seeing a revision of this interesting and innovative article.

Best regards,

Brenda McCowan

Preprint DOI: https://www.biorxiv.org/content/10.1101/2021.02.21.432190v1

\section{Reviewed by Krishna Balasubramaniam, 2021-03-23 20:14}

Download the review (PDF file)

\section{Reviewed by anonymous reviewer, 2021-03-19 22:17}

This study compares an empirical data set on a feral population of horses with simulated data from multiple agent based models to ask whether behavioral synchronization in a multilevel society can be described by a simple model, and whether this model must include the network of social relationships to accurately describe the pattern of synchronization observed. I think the study is well-designed, the hypotheses behind the different models make sense, and the measures of behavioral synchrony (e.g., number of individuals switching from one state to the other) are appropriate. Some aspects of the sampling process for the empirical data could be clarified a little more (see specific line comments below). For instance, behavioral data were obtained using aerial drones which took photo or video scans of the entire herd, and it is unclear whether a validation process was done to verify that e.g., a horse described as 'resting' in the scan image was truly resting according to an observer on the ground. I really like the inclusion of a social network into the agent-based models to investigate the potential influence of social relationships on behavioral synchrony. I think those familiar with network analysis would like to see additional information about these networks, such as density of the association networks generated for each scan observation or how many components were in each network (if not a single connected component). Finally, there are grammatical errors throughout the paper that cause some confusion about the authors' meaning, particularly in the Discussion section, which could benefit from revision. I have made some suggested revisions in the line comments below, but this was not possible for all such occurrences.

Line 120: Please explain further the definition and calculation of 'zones'. Because I am unfamiliar with such analyses, I am a little concerned that when the authors say that they calculated "...distances between all pairs of individuals in the same zone" there are some pairs that occur in different 'zones' yet their inter-individual distance would still be relevant to calculate. A definition of zones could potentially clarify this.

Line 121: Were different times of day over-sampled relative to other times of day? Although the Supplementary files show that an analysis was done to see if horses' behavior differed according to time of day, the additional information about level of sampling per 30-minute interval would also provide helpful context.

Line 131-132: I don't quite understand what was done with the histogram of the inter-individual distance data. What is meant by "...from the distance data to the shape under the R environment"? My guess is this examination of the data was performed in R or RStudio (the 'R environment'), but it seems like some words are missing from this sentence.

Lines 133-135: Suggest revising to read: "As shown in Figure 2, the histogram of inter-individual distances had two peaks - at the $2 \mathrm{nd}$ bin $(0.9-1.8 \mathrm{~m})$ and at the $55 \mathrm{th}$ bin $(49.7-50.6 \mathrm{~m})$ with a bin-width of $0.92 \mathrm{~m}$. The minimum frequency, or nadir, between these two peaks was observed at the 12th bin (10.1-11.0m), and we selected this as the threshold distance between...." 


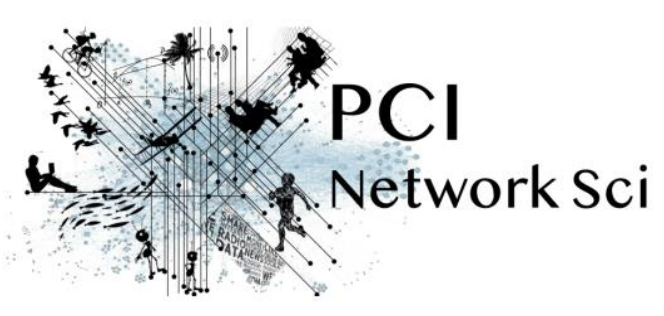

Lines 140-142: The word 'scaled' doesn't seem quite right here. Perhaps 'assigned'? Pairs of horses whose inter-individual distance was smaller than 11 meters were assigned an edge weight of 1, based upon the threshold distance defined above. All other pairs were assigned an edge weight of 0 .

Line 143: What is an 'unconnected component'? Is this a typo and the authors meant to write 'connected component' or is there another concept being applied here? Also, the authors say that pairs of individuals were considered to be associated when they were part of the same unconnected component. What is the different between being 'associated' and identifying 'social relationships'? Is being 'associated' only needed to determine which individuals in the herd were isolates in the network graph, and it should not be conflated with what defines an edge in the network?

Line 156: Shouldn't this say “...in our case, a scan every 30 minutes..."? Because a single scan was not 30 minutes in duration, but spaced 30 minutes apart. The duration of the (drone) scans is noted to be 4 minutes and 24 seconds on average.

Lines 115-118 and 159-164: Did the authors validate that their assignment of resting vs. moving, as captured in the drone photographs, was correct?

Line 159: How many of the scans/ observations did not include $90 \%$ of the units (and were thus omitted from analysis)?

Lines 164-167: I don't fully understand how these phases were defined. Please revise these sentences.

Line 178: Please define refractory period for readers.

Line 310: Revise to read: "...how many individuals changed state..."

Line 313: Revise to read: “...using linear regression in the R environment.”

Lines 321-322: Revise to read: “Mantel tests were performed using the R package 'vegan', and K-S tests were performed using the function 'ks.test' in the R environment."

Lines 323-324: Revise grammar; for instance: “...horses live in a multilevel society and are therefore expected to show social cohesion and behavioral synchronization."

Lines 355-359: Revise grammar, for instance: "Figure 6 shows the comparison between model-generated synchronization scores and synchronization scores from the empirical data. The model simulations that did not consider social relationships (i.e., independent, absolute anonymous, and proportional anonymous models) showed a lot of overlap in the histograms of intra-unit and inter-unit synchronization scores, unlike the observed data which show clear separation between intra and inter-unit synchronization scores (Figure 6)." In addition, it would help to better incorporate Mantel test and K-S test results (in Table 2) at the appropriate comparisons in the text to better integrate Table 2 information with results descriptions.

Lines 370-372: Maybe rephrase a little here. Perhaps something like “...better matched the empirical data than the null model (model A), whereas..." The phrasing 'showed better results' is a little imprecise.

Line 385: I assume you are comparing the observational data to the model simulation data when you say "The observation data had higher intra- and inter-unit synchronization..." but this should be made clear, and if so, are you referring to ALL models? However, I am not sure what is being compared in the following lines (i.e., "number of individuals changes state..." is higher than what?)

Lines 385-400: Grammatical revisions are needed to clarify what discussion points and conclusions are being made. This paragraph is confusing as it is currently written.

Line 406: Could this not have been examined in the agent based models? Can the parameter settings, or the design of the model for agents' behavior, be changed to allow the agents to 'see' only the other units that are closest to them, such as implementing a certain distance radius around an agent? 


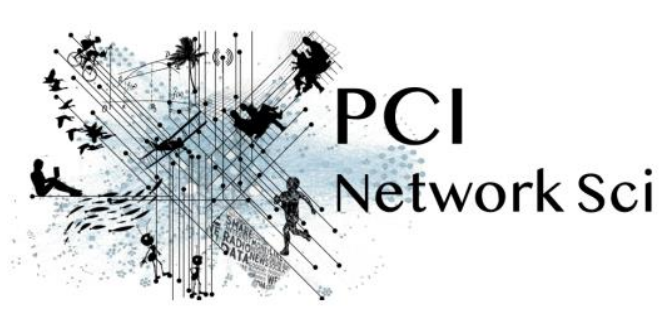

\section{Reviewed by Frédéric Amblard, 2021-03-23 13:10}

The authors propose a very interesting approach using a multi-agent model tested on several underlying social networks in order to identify the social structure that is most likely driving synchronization behaviour in feral horses. The hypotheses taken to generate social networks and the different aspects of the model are well grounded as well as the data anlysis conducted to analyse the correspondence between the models results and the empirical data. Those latter, based on drone observations are particularly promissing (despite the "low" autonomy of the drone) and the results are quite convincing.

The few remarks (below) I have are rather a consequence the inspirations $i$ had reading the paper, rather than real criticisms about the approach and the methodology that are very convincing to me.

A first remark concerns the term synchronization, as having in mind models like the synchronization of fireflies it is maybe a bit misleading and the current case corresponds rather to a more global case of social influence. But if the term is the real one used in this particular field, I would totally accept it could be a seen as a synchronized process at a larger time scale.

A second one is rather a perspective about the global method concerning the generation of social networks that is quite classical in the field of animal networks study. The point is that using spatial distance to generate networks you can not really distinguish in between a spatial influence and a social one. This is not totally the casee there as the different social networks tested enable to fill a gap in between the two options. However, one possible solution (and it is maybe the one tested there but it is not totally clear) would be to use half of the dataset in order to generate the social network and once generated to use the other half in order to test the different hypotheses. Such option would be in particular interesting when using directed relationships rather than bidirectional ones, trying to capture a single sided influence especially introducing the perception of the individuals that is oriented (therefore a symetric relation would correspond to the case where two horses are standing face to face).

A complementary approach that could potentially shed a different light on the system concerns the analysis of the evolving social network in particular concerning the evolution of the social communities identified along time. 